\title{
Ist die Sprache ein selbständiger Faktor der Wissenschaftsgeschichte?
}

\author{
Über die Anstößigkeit unerwarteter Sprache [1990/1994]
}

Hugo Steger zum 60. Geburtstag

Ist die Sprache ein selbständiger Faktor der Wissenschaftsgeschichte?

Die Frage birgt ihre Schwierigkeiten. Sprache ist den einen bloßes Instrument, durchsichtig auf den Gegenstand hin wie Wasser, anderen scheint sie an seiner Bildung Anteil zu haben. Sie ist die anpassungsfähigste soziale Institution, die flexibelste. Vielleicht ist sie darum auch die unauffälligste, selbstverständlich verfügbar und kaum ins Blickfeld geratend.

Wenn Sie eine improvisierte Beobachtung zur Vortragspraxis im Saal der Mainzer Akademie erlauben:

Der naturwissenschaftliche Flügel spricht fast immer frei; es gehört anscheinend zur Spielregel, nicht abzulesen, und zwar nicht nur dann, wenn mit Schaubildern und Wandtafel gearbeitet wird. Die Sache stellt sich durch das eine oder das andere Wort von selber dar. Das erinnert an den Ausspruch des berühmten Kollegen im gotischen Zimmer: „Ich kann das Wort so hoch unmöglich schätzen.“ (Goethe, Faust: Vs 1226)

Der geisteswissenschaftliche und sozialwissenschaftliche Flügel scheint der fixierten Formulierung viel mehr Gewicht zu geben, er liest häufiger ab, hält sich ans Manuskript, es sieht so aus, als habe die Sprache ein Eigengewicht, als sei sie am Aufbau der Gegenstände und an ihrer Wirkung beteiligt und dies beides kaum $\mathrm{zu}$ trennen.

Das kleine Häuflein zwischen den großen Klassen, der Tisch der Schriftsteller, bindet sich meist ganz ans Wort; es erscheint als die Hauptsache. Das Wort ist für manchen das einzige, was in Betracht kommt. Wir finden hier die Ansicht, die Wirklichkeit sei sprachgemacht, und was das eigene Tun angehe, so gelte:

Eine Wirklichkeit ist nicht vonnöten

ja es gibt sie garnicht, wenn ein Mann

aus dem Urmotiv der Flairs und Flöten

seine Existenz beweisen kann.

(Benn, Wirklichkeit)

Die Frage, ,Ist die Sprache ein selbständiger Faktor der Wissenschaftsgeschichte?', geht in diesem Fall vom Katzentisch aus, von der Vermutung eines erheb- 
lichen Gewichts der Sprache, aber mein Beobachtungsgegenstand ist überwiegend die Geschichte der Naturwissenschaft.

In der Geschichte der Wissenschaften passiert es immer wieder, dass ein Autor sich neu, anders, vielleicht auch unmittelbarer gegen die Sachen stellt. In solchen Fällen wird die überlieferte Sprache als Institution sichtbar, fühlbar. Sie wehrt sich und erscheint als gar nicht mehr so beweglich. Zur Geschichte der Wissenschaft gehört fortwährend ein Überhang institutionalisierter Sprache. Als schriftlich fixiertes Gebilde kann sie die Trägheit der Institution annehmen.

Wie lässt sich unserer Frage beikommen? Ich möchte sie hier aus nur einem Blickwinkel angehen, nicht allgemein und philosophisch, sondern sozialpsychologisch.

Ich schlage vor, das Stirnrunzeln zum Ausgangspunkt einer Geschichte der Wissenschaftssprache zu wählen. Das Stirnrunzeln ist bekanntlich eine der Gesten, durch die wir in mündlichen Situationen einen befremdlichen Sprachgebrauch ahnden. Andere Formen der Sanktion sind, versteckt zu lächeln oder sich zu räuspern, offen zu lachen, verschlossen schweigend vor sich hinzusehen oder einfach wegzuhören. Ziemlich häufig ist die spottende Nachahmung störender Sprache, am seltensten vielleicht ihre ausdrückliche, begründete Ablehnung.

Etwas Vergleichbares lässt sich in der Geschichte der Wissenschaften beobachten. Man stößt immer wieder auf den Fall, dass ein Sprachgebrauch Anstoß erregt, einen Skandal hervorruft, Sanktionen auslöst und jedenfalls Wirkung ruckartig fördert oder verhindert.

Diese Fälle sind für den Sprachhistoriker deshalb so interessant, weil, wenn ein Regelverstoß geahndet wird, erkennbar wird, was als Regel gilt. Die Sanktionen sind ein Indikator geltender Normen, wir können sie geradezu als Instrument verwenden, um Sprachgebrauchsnormen aufzudecken. Im Folgenden versuche ich, das Gesagte an vier Kreisen sichtbar zu machen. Ich hebe vier Sprachgebrauchstraditionen hervor, mit denen im Lauf unserer Wissenschaftsgeschichte gebrochen wird. Die Themen sind:

1. Die Anstößigkeit des Deutschen, wo Latein erwartet wird.

2. Die Anstößigkeit von Poesie und Rhetorik, wo Wissenschaft erwartet wird.

3. Die Anstößigkeit bildungssprachlicher Spekulation, wo Empirie und Mathematik erwartet wird.

4. Die Anstößigkeit des Deutschen, wo Englisch erwartet wird. 


\section{Die Anstößigkeit des Deutschen, wo Latein erwartet wird}

Der Schritt vom Lateinischen zum Deutschen ist derjenige Regelverstoß, der die heftigsten Reaktionen auslöst, zu Recht, es ist ein revolutionärer Akt. Der Wechsel ist nicht gleichbedeutend mit der Frage, ob Wissenschaft in einer universalen Fremdsprache oder in der heimischen Muttersprache gelehrt werden soll, wie es einer nationalen Sprachgeschichtsschreibung erscheinen konnte. Paracelsus und später Thomasius ist zwar schon von Zeitgenossen vorgehalten worden, sie beherrschten halt das Lateinische nicht genug, aber der Vorwurf war gegenstandslos, Latein und Deutsch waren ihnen mündlich und schriftlich vollständig geläufig, zwei Möglichkeiten des Sprachgebrauchs, zwischen denen sie hin und her pendelten. Die Frage war, in welchem sozialen Umfeld Wissenschaft getrieben und verbreitet werden sollte.

Paracelsus in Basel $^{1}$ ist ein Fall, der bereits alle Dimensionen dieses Übergangs sichtbar macht. Er tritt im März 1527 das alte Amt eines Stadtarztes von Basel an und gerät sofort in das Spannungsfeld zwischen Universität und Stadt. Der Magistrat der Stadt hat ihn in das Amt des physicus berufen, und es ist unstrittig, dass ihm damit das Recht übertragen ist, an der Universität medizinische Vorlesungen $\mathrm{zu}$ halten. Aber wie weit untersteht er damit auch dem Aufsichtsrecht der Universität?

Er liest zunächst auf lateinisch, beginnt dann parallel auf deutsch eine chirurgische Vorlesung (vgl. Paracelsus 1922-1933, I, 4: XXIIf.), die über den Kreis der eigentlichen Ärzte hinaus auch die Wundärzte anspricht, Leute, die auf dem Weg über eine Lehre und ohne Latein zu ihrer Profession gelangen. Der Kreis seiner Hörer erweitert sich.

Darauf versucht er, eine gründlichere Reformation der Medizin zu initiieren, indem er dem den Kampf ansagt, was man als das jahrhundertealte Konnotat, den Assoziationshof lateinisch gefasster und geronnener Wissenschaft bezeichnen könnte: der Autorität der Alten und der an einen schriftlich überlieferten Kanon gehefteten Wahrheitsvermutung. In einer Intimatio vom 5. Juni 1527, einem Vorlesungsprogramm, das er als Flugblatt verbreitet - (üblicherweise wird es ans Schwarze Brett angeschlagen) - schreibt er auf lateinisch:

\footnotetext{
1 Zum Folgenden vgl. Hodermann 1891: 7ff.; Sudhoff 1936: 24-45; Peuckert 1944: 140ff., 151ff., 161ff.; Blaser 1979, bes. die Kap. „Neue Erkenntnisse zur Basler Zeit des Paracelsus“, 20-81, sowie „,Manes Galeni adversus Theophrastum'. Ein Beitrag zur Deutung des Basler Pasquilles gegen Paracelsus“, 82-102, „,Amplo stipendio invitatus‘. Zur Frage der Stellung und Besoldung des Paracelsus in Basel“, 103-114. Vgl. auch Pörksen 1986a: 45ff.
} 
Quis enim nescit [...] - Wer weiß es denn nicht, daß die meisten Ärzte heutiger Zeit zum größten Schaden der Kranken in übelster Weise daneben gegriffen haben, da sie allzu sklavisch am Worte des Hippokrates, Galenos und Avicenna und anderer geklebt haben, als ob diese wie Orakel aus dem Dreifuß des Apoll herausklängen, von deren Wortlaut man auch nicht um Fingers Breite abweichen dürfte. (Übersetzung nach Sudhoff 1936: 28f.)

Er verlagert den Wahrheitsbeweis von der Autorität der Alten und von der überlieferten Schrift auf die Natur der Dinge und auf Erfahrung, Arbeit und eigene Erwägung, rei natura, experientia, labor, ratio.

Am 24. Juni wirft er öffentlich ein medizinisches Kompendium ins Johannisfeuer. Die Sanktionen ließen nicht auf sich warten. Die Universität sucht seine Kollegs zu hintertreiben, verbietet ihm den Hörsaal, zieht die Rechtmäßigkeit seines Doktortitels in Frage und zwingt ihn zu seiner Beeidung, entzieht ihm das Promotionsrecht. Er wendet sich in einer Beschwerde an den Magistrat:

also das ir min obern herren, decanen und facultet (und nit die ienigen) sigen, uf das ich billich mög als ein ordinarius promoviren in doctores. (Zit. nach Sudhoff 1936: 32)

Nicht lange danach ist eines Sonntagmorgens an den Türen des Doms, der Kirchen zu St. Martin und St. Peter und an der neuen Burse ein Pasquill in Versen angeschlagen, unterzeichnet „Ex inferis“, „Aus der Unterwelt“, überschrieben „Manes Galeni adversus Theophrastum, sed potius Cacophrastum“, „Die Manen Galens gegen Theophrastus oder besser Cacophrastus“. Er wird als Windbeutel und Dieb gescholten, seine eigenwilligen Termini werden parodiert.

Dispeream si tu Hippocrati portare matellam Dignus es, aut porcos pascere, Vappa, meos. Verrecken will ich, wenn Du würdig bist, dem Hippokrates das Nachtgeschirr nachzutragen, oder meine Schweine zu hüten, Du Lappes. (Zit. nach Sudhoff 1936: 40)

Das Gedicht ist vermutlich von drei Hörern des Paracelsus verfasst, aber von der Universität angestiftet worden. Paracelsus konnte sich nur elf Monate in Basel halten.

Die Frage war, wie gesagt, in welchem sozialen Umfeld Wissenschaft getrieben und verbreitet werden sollte. Nicht ein Abstraktum ,Sprache“ stand zur Diskussion, sondern eine Entscheidung zwischen zwei grundverschiedenen Kulturen: zwei Spielregeln der Wahrheitsfindung und zwei Öffentlichkeiten, Trennung von Universität und Stadt oder Durchdringung der Stadt mit Wissenschaft. $^{2}$

2 Vgl. die Öffnung der Universität von Florenz für die Bürger der Stadt durch Cosimo I., Pörksen 1986a: 56f. 
Die Niederlegung der lateinischen Trennmauer führt dazu, dass Wissenschaft das öffentliche Weltbild einer Kritik unterzieht und zugleich sich selbst dem Zugriff derer aussetzt, die über dieses Weltbild die Oberaufsicht beanspruchen. Latein konnte ein Schutzwall sein. Galileo Galileis Sidereus Nuncius, der seine bedeutendsten astronomischen Entdeckungen veröffentlichte, erschien $1610 \mathrm{mit}$ Zustimmung zweier Inquisitoren. Als wenig später seine Discorsi die gleichen Inhalte in der Landessprache und ihrem Duktus mitteilen, tritt die Inquisition in Aktion (vgl. Olschki 1922: 63f., 66f.). In der Sprache der Allgemeinheit ruft die neue Wahrheit ihren Rivalen auf den Plan.

160 Jahre nach Paracelsus, zur Ostermesse 1687, hat Christian Thomasius am Schwarzen Brett der Leipziger Universität auf deutsch zu einem Kolleg über Graciáns Handorakel der Weltklugheit eingeladen. Sprache und Thema hängen offensichtlich zusammen. Er unterrichtete nicht im Talar, sondern in der Kleidung der Stadt, in Zivil, und die Vorbereitung der Studenten auf die praktische Welt erscheint ihm zunehmend als Aufgabe. Seine gezielte Provokation richtet sich wie die seines Vorgängers in Basel gegen das Schriftprinzip, die Versteinerung der Überlieferung durch ihr Weitergetragenwerden in dem konservierenden lateinischen Kanon, das „aristotelische Orgelwerk“, und er verbindet sie mit einer Neubestimmung des Bildungsauftrags der Universität. Auch Thomasius konnte sich nicht halten, er entwich von Leipzig nach Halle, an seinen dortigen Namen knüpft sich dann aber der Durchbruch der deutschen Sprache an der Universität (vgl. Hodermann 1891: 7ff.; Pörksen 1986a: 45ff.).

Nachdem die soziale Norm durchstoßen, der Schritt von dem einen sozialen Umfeld in das andere geschehen ist, kann die Frage Fremdsprache oder Muttersprache in den Vordergrund rücken. In einer biographischen Skizze über den Mathematiker Gauß, der noch im 19. Jahrhundert lateinisch schrieb, bemerkt der Engländer Eric T. Bell 1937:

An Stelle des leichten Lateins, das Euler und Gauß genügte und das in wenigen Wochen erlernbar war, muß nun jeder Wissenschaftler zu seiner eigenen Sprache noch zwei oder drei andere mindestens lesen lernen. Gauß wehrte sich, solange er konnte, aber schließlich mußte auch er nachgeben und seine astronomischen Werke deutsch schreiben. (Bell 1967: 225)

\section{Die Anstößigkeit von Poesie und Rhetorik, wo Wissenschaft erwartet wird}

Die Wissenschaft trennte sich nicht nur in einem sehr allmählichen, stockenden, schwankenden Prozess von der Autorität der europäischen gelehrten Universal- 
sprache, sie verabschiedete sich auch von der Vorstellung, die der Humanismus zunächst belebt hatte, dass poetische und rhetorisch geprägte Formen gleichberechtigte und ernst zu nehmende Gattungen wissenschaftlicher Mitteilung seien. Ein zeitgenössischer Historiker der Royal Society in London, Thomas Sprat, schreibt 1667, die neue Akademie

verpflichtete ihre Mitglieder auf einen präzisen, nüchternen, ungezwungenen Stil, auf konkrete Ausdrücke, klare Bedeutungen und eine natürliche Leichtigkeit, die alles so weit wie möglich der mathematischen Klarheit annähert und sich lieber der Sprache der Handwerker, Bauern und Kaufleute bedient als der der geistreichen Herren und Gelehrten (nach Heidelberger \& Thiessen 1981: 243).

Der neue Stil ist interessanterweise auch hier an ein neues soziales Feld gebunden.

Dieses Stilideal wurde zu einem zweiten Stolperstein in der Geschichte der Wissenschaftssprache. Zwar wurde im 18. Jahrhundert Newtons Optik auch noch in der Form von Sonetten ausgearbeitet (vgl. Heidelberger \& Thiessen 1981: 248), und Goethe, der um 1780 einen Roman des Weltalls plante, hat seine Einsichten in die Metamorphose der Pflanzen und Tiere nicht nur in Aufsätzen, sondern auch in der traditionellen Form der Elegie, in Distichen, niedergelegt. Aber das naturwissenschaftliche Lehrgedicht war ihm selbst nicht mehr unproblematisch, und er erfuhr das 18. Jahrhundert als Epoche der Trennung und sprachlichen Aufsplitterung der Fächer:

Vereinzelt behandelte man sämtliche Fähigkeiten; Wissenschaften und Künste, Geschäftsführung, Handwerk und was man sich denken mag, bewegte sich im abgeschlossenen Kreise. [...] Poesie und Wissenschaft erschienen als die größten Widersacher. Indem sich nun jeder einzelne Wirkungskreis absonderte, zersplitterte sich auch in jedem Kreise die Behandlung. (LA I, 9: 106)

Goethe empfand die Aufnahme seiner Metamorphose der Pflanzen von 1790 als „kalt, fast unfreundlich“, was angesichts des tatsächlichen Befundes einen etwas hypochondrischen Eindruck macht ${ }^{3}$ und vielleicht erst erklärlich wird, wenn man überlegt, dass er seinem naturwissenschaftlichen Werk zunehmend größere Bedeutung zumaß als seiner künstlerischen Hinterlassenschaft. Aber das Gefühl des Misserfolgs wird produktiv, es führt ihn zu interessanten Erklärungsversuchen.

Der Aufsatz über Die Metamorphose der Pflanzen lag nach Goethes Eindruck in dreierlei Hinsicht quer zur eingeübten Erwartung des Publikums:

3 LA I, 9: 11; vgl. Goethe 1984: 137; vgl. auch die Rezensionen der Metamorphose der Pflanzen, LA II A: passim. 


\section{Auf dem Gebiet der Botanik herrschte ein anderer Diskurs.}

Sie antworteten alle mehr oder weniger in Bonnets Redensarten: denn seine Kontemplation der Natur hatte, durch scheinbare Faßlichkeit, die Geister gewonnen, und eine Sprache in Gang gebracht, in der man etwas zu sagen, sich untereinander zu verstehen glaubte. $\mathrm{Zu}$ meiner Art mich auszudrücken wollte sich niemand bequemen. (LA I, 9: 69f.)

2. Der Dichter fällt durch seine wissenschaftliche Arbeit aus seiner „Rolle“. Die durch seine bisherigen Arbeiten geschaffene Erwartung wirkt zurück als Rahmen, in dem er sich zu halten hat. Göschen lehnt den Aufsatz ab, das Publikum „stutzt“. Der „Übergang in ein anderes Feld“ wird beim Publikum nicht gern gesehen, „denn nach seinem Wunsch, sich gut und gleichförmig bedient zu sehen, verlangt es an jeden, daß er in seinem Fache bleibe [...]“ (LA I, 9: 64).

3. Das Publikum fasst den Autor in dem ihm bisher gewohnten Rahmen auf, sein wissenschaftliches Werk wird missverstanden als Werk der Poesie. Ein Leser beruhigt sich, wie Goethe mitteilt, weil der Dichter die Sache „nach Ovids Weise genommen“ (LA I, 9: 106). - Das ästhetische Missverständnis, das insofern naheliegen konnte, als Goethe eine Verbindung von Wissenschaft und Poesie anstrebte, gehört zur Geschichte der Aufnahme seines Werks.

In allen drei Fällen ist es eigentlich die Apperzeptionsgewohnheit, die Gewöhnung an die Sprache einer wissenschaftlichen Schule oder an das Bild eines Autors, was eine Aufnahme von Andersartigem verhindert oder überblendet.

Alexander von Humboldt, dessen Laufbahn als Naturforscher am nachhaltigsten von Goethe initiiert worden ist, geht zu Beginn des 19. Jahrhunderts bereits davon aus, dass Wissenschaft und Poesie Widersacher seien. Er übergibt 1808 den ersten Niederschlag seiner Weltreise, die später so erfolgreichen Ansichten der Natur, „schüchtern“ dem Publikum, spricht von Verirrungen und einem Mangel an Haltung. „Das Gefühl und die Phantasie ansprechend, artet der Stil leicht in eine dichterische Prosa aus“ (Humboldt, A. von 1969: 5). Tatsächlich liest man in dem Kapitel „Über die Wasserfälle des Orinoco“ nach einer Reihe exakter Angaben:

Dichter Nebel schwebt ewig über dem Wasserspiegel. Durch die dampfende Schaumwolke dringen die Gipfel der hohen Palmen. Wenn sich im feuchten Dufte der Strahl der glühenden Abendsonne bricht, so beginnt ein optischer Zauber. Farbige Bögen verschwinden und kehren wieder. Ein Spiel der Lüfte, schwankt das ätherische Bild. (Humboldt, A. von 1969: 47)

Der Versfuß des Hexameters grundiert nicht nur an dieser Stelle seine wissenschaftliche Prosa. Das Werk ist aufgespalten in große epische Naturgemälde, die - seiner Vorrede zum Trotz - mit aller List der Komposition angelegt sind und den gewünschten „Totaleindruck“ vermitteln, und in den weit umfangreicheren 
Anmerkungsteil, der Nachweise, Tabellen und Zahlen, ganze Konvolute harter Daten enthält. Poesie und Wissenschaft, die beiden konträren Zugänge zur Wirklichkeit, treten hier also auch äußerlich auseinander. In der zweiten Auflage von 1826 findet er den Mut, seine Erzählung Die Lebenskraft oder der rhodische Genius, die Schiller 1795 in die Horen aufgenommen hatte, wieder abdrucken zu lassen, in der dritten Auflage von 1849 zitiert er eine briefliche Bemerkung seines verstorbenen Bruders Wilhelm zum „rhodischen Genius“:

Die Entwicklung einer physiologischen Idee ist der Zweck des ganzen Aufsatzes. Man liebte in der Zeit, in welcher derselbe geschrieben ist, mehr, als man jetzt tun würde, solche halbdichterische Einkleidungen ernsthafter Wahrheiten.

Auch Alexander von Humboldt nennt die Erzählung nun einen „Aufsatz“, was sie nicht ist, und hebt hervor, dass er in dieser Auflage die wissenschaftlichen Erläuterungen auf den neuesten Stand gebracht habe, in der Hoffnung, die „Wichtigkeit genauer numerischer Angaben“ werde erkannt und dem ,dogmatischen Halbwissen“ der „sogenannten höheren Kreise geselligen Lebens“ gesteuert. „Die Verbindung eines literarischen und eines rein szientifischen Zweckes, der Wunsch, gleichzeitig die Phantasie zu beschäftigen und durch Vermehrung des Wissens das Leben mit Ideen zu bereichern“, von der 1849 die Rede ist, charakterisiert auch sein episches Alterswerk, den Kosmos (Humboldt, A. von 1969: 7f.).

Diese Verbindung wird zunehmend unmöglich. W. H. Riehl, der Volkskundler, hat sie noch um die Jahrhundertmitte gesucht, er sprach von einer „Doppelkunst““, aber zeitnäher war doch wohl die traditionsreiche Unruhe, mit der Freud 1895 an einer berühmt gewordenen Stelle seine Studien zur Hysterie unterbricht:

Ich bin nicht immer Psychotherapeut gewesen, sondern bin bei Lokaldiagnosen und Elektroprognostik erzogen worden wie andere Neurophathologen, und es berührt mich selbst noch eigentümlich, daß die Krankengeschichten, die ich schreibe, wie Novellen zu lesen sind, und daß sie sozusagen des ernsten Gepräges der Wissenschaftlichkeit entbehren. Ich muß mich damit trösten, daß für dieses Ergebnis die Natur des Gegenstandes offenbar eher verantwortlich zu machen ist als meine Vorliebe; [...]. (Freud 1940ff., I: 227)

Auf der einen Seite also „Lokaldiagnosen“ und „Elektroprognostik“, kurz „Neuropathologie“, auf der anderen Seite „Ich bin“, „Es berührt mich“, „wie Novellen zu lesen“. Freud benennt hier nicht nur den Zwiespalt zwischen den beiden grund-

4 Vgl. Lepenies 1985: 241f. Der Reputationsverlust des schriftstellernden Gelehrten ist nicht nur ein Thema des Kapitels über Riehl, sondern wird in den verschiedensten Zusammenhängen angeschlagen. 
verschiedenen Gattungen, sondern er führt auch jede in der ihr eigenen Sprache vor und stellt seinen Zwiespalt dar. Die Antwort, die er an dieser Stelle findet, charakterisiert später in ausgearbeiteter Gestalt sein gesamtes Werk, er wechselt und verschränkt in ihm die beiden Stile, den des einfühlenden, die seelischen Vorgänge beweglich und phantasievoll darstellenden Schriftstellers und den des distanzierten, kühlen, objektiven Naturwissenschaftlers, der durch seine biologische, chemische, physikalische Bildersprache einen scheinbaren Anschluss an die Leitdisziplinen seiner Epoche herstellt. - Das schlechte Gewissen, die im Eingeständnis der Unruhe vorweggenommene Sanktion der wissenschaftlichen Öffentlichkeit, hat also in seinem wie in Humboldts Fall beträchtliche Folgen für die Form des Werks. ${ }^{5}$

Die Grenze zwischen den beiden Widersachern Wissenschaft und Poesie ist seit den siebziger Jahren wieder löchrig geworden. Während Watson mit seinem Buch The Double Helix von 1968, das die Geschichte der Entdeckung der DNSStruktur in der spannendsten Weise erzählt, in der naturwissenschaftlichen Welt zunächst noch einen Skandal hervorrief (vgl. Weinrich 1986b: 188ff.), hat der Schlachtruf „narratio rediviva!“ in den Sozialwissenschaften und Geisteswissenschaften beträchtliche Folgen gehabt, und auch die Naturwissenschaften öffnen sich, scheint es, dem Widersacher.

\section{Die Anstößigkeit bildungssprachlicher Spekulation, wo Empirie und Mathematik erwartet wird}

Nach 1830, Hegels Todesjahr, wird an der Berliner Universität die Sprache der Naturphilosophie zunehmend verdächtig. Johannes Müller, Inhaber des berühmten ersten Lehrstuhls für Physiologie in Berlin, an dem durch seine Schüler, vor allem durch Du Bois-Reymond und Helmholtz, das moderne naturwissenschaftliche Weltbild, sein Physikalismus und Materialismus, in scharfer Reaktion auf

5 Vgl. die „Vorbemerkung“ zur ersten Ausgabe der Traumdeutung: „Mit der Mitteilung meiner eigenen Träume aber erwies sich als untrennbar verbunden, daß ich von den Intimitäten meines psychischen Lebens fremden Einblicken mehr eröffnete, als mir lieb sein konnte und als sonst einem Autor, der nicht Poet, sondern Naturforscher ist, zur Aufgabe fällt" (Freud 1940ff., II/III: VIII). - Der Schluss der Vorbemerkung enthält vielleicht einen Anklang an Goethes Werther. Wie Goethe in seiner Herausgebereinleitung hofft, man werde dem Schicksal des armen Werther die „Thränen nicht versagen“, so erhofft sich Freud, das die von seinem Werk betroffenen Personen „wenigstens dem Traumleben Gedankenfreiheit nicht werden versagen wollen“. 
Hegel und die Jenaer Romantik, auf die deutsche Naturphilosophie vom ersten Jahrhundertdrittel insgesamt, ausformuliert wurde, ${ }^{6}$ könnte dafür als erstes Beispiel dienen.

Er begann um 1820 als Naturphilosoph, schloss sich danach in seinen Studien zur Physiologie des Gesichtsinnes zeitweise Goethe an und wurde seit seiner Bildungsgeschichte der Genitalien von 1830 bis zu seinem Tod 1858 mit 20 Schriften und 250 größeren und kleineren Abhandlungen $\mathrm{zu}$ einem der maßgeblichen Erfahrungswissenschaftler in Deutschland. Seine naturphilosophischen Anfänge erschienen ihm im Rückblick nur noch als „Sprache“.

$\mathrm{Du}$ Bois-Reymond berichtet darüber in seiner schönen Gedächtnisrede, die frühen Arbeiten verrieten bereits alle Sorgfalt seines Lehrers.

\begin{abstract}
Zugleich aber zeigen sie uns, ein bemerkenswerter Umstand, den jugendlichen Johannes Müller gänzlich versunken in dem Traummeer jener mit polaren Gegensätzen spielenden falschen Philosophie der Natur, die während des ersten Viertels dieses Jahrhunderts der deutschen Wissenschaft tiefere Wunden schlug als aller Kriegslärm des westlichen Eroberers. Das Leben in der Bewegung ist ihm „eine organische Säule; die Pole sind Beugung und Streckung, oder die Kreisbewegung und die Bewegung in der Längenform: - beide auseinander gerissene Hälften der parabolischen Linie, auf welcher das Leben spielt“ (Isis von Oken, 1822, 1. Bd., 1. Heft, S. 61). Mit solchem Ingrimm blickte Müller nachmals auf diese Verirrungen zurück, daß er selber dieser Arbeiten nie wieder gedachte, und jedes Exemplar derselben, dessen er habhaft werden konnte, aufkaufte und verbrannte. (Du Bois-Reymond 1860: 34)
\end{abstract}

Selbst Hermann von Helmholtz, der zusammen mit Du Bois-Reymond, Brücke und Ludwig 1847 ein Manifest formulierte, durch das sie „die Physiologie auf eine chemisch-physikalische Grundlage stellen und ihr einen gleichen wissenschaftlichen Rang mit der Physik geben“ wollten (Mendelsohn 1974: 253), geriet mit seiner berühmtesten Arbeit aus dem gleichen Jahr, der Abhandlung Über die Erhaltung der Kraft, in den Verdacht philosophischer Spekulation. Die jüngeren Physiker und Physiologen stimmten der neuen Theorie bei, die älteren lehnten sie fast sämtlich ab. „Sie waren geneigt, die Richtigkeit des Gesetzes zu leugnen und in dem eifrigen Kampfe gegen Hegels Naturphilosophie, den sie führten, auch meine Arbeit für eine phantastische Spekulation zu erklären, “schrieb er im Rückblick (Helmholtz \& Du Bois-Reymond 1986: 20). Wir werden sehen: die eingefahrene Sprache überblendet auch hier Rezeption.

Er trug dieses berühmte Dokument 1847 in der Berliner Physikalischen Gesellschaft vor, ein 26jähriger Medizinstudent, der sich vorher mit Tierwärme beschäftigt hatte und, während er an Entwürfen zu dieser Abhandlung schrieb, Dienst am

6 Vgl. den ungemein aufschlussreichen Aufsatz von Everett Mendelsohn 1974: 251. 
Militärhospital in Potsdam tat. Aus einem Brief vom 12. Februar 1847 an Du BoisReymond wissen wir, dass er sich alle Mühe gab, die Sprache zu finden, die vor der Physikalischen Gesellschaft Gnade finden würde (Helmholtz \& Du Bois-Reymond 1986: 78f.):

Lieber Freund, ich übersende Dir beiliegend meinen Versuch einer Einleitung zur Konstanz der Kraft, nicht weil ich damit fertig zu sein glaube, denn ich habe beim Durchlesen gesehen, daß vielleicht nichts darin bleiben kann, sondern weil ich noch nicht absehe, wie oft ich ihn noch umarbeiten muß, ehe er fertig ist, und weil ich zu erfahren wünsche, ob $\mathrm{Du}$ die Art der Darlegung für eine solche hältst, die bei Physikern Eingang finden kann. Ich habe mich bei der letzten Ausarbeitung zusammengenommen und alles über Bord geworfen, was nach Philosophie roch, soweit es nicht dringend nötig war, [...].

Trotz der wiederholten Versuche, den Aufsatz an die Sprache der Physik anzugleichen, wurde er von dem Jahresorgan der Physikalischen Gesellschaft als nicht ausreichend experimentell abgelehnt, und aus einem Brief Du Bois-Reymonds vom 4. August 1847 lässt sich entnehmen, welche subtilen Überlegungen die Freunde anstellten, bis Helmholtz dieses herausragende Dokument der naturwissenschaftlichen Moderne auf eigene Hand veröffentlichte (Helmholtz \& Du BoisReymond 1986: 82f.; vgl. Mendelsohn 1974: 257).

Der Fall Mendel kann als Parallel- und Gegenbeispiel dienen. Johann Mendel (1822-1884), der nach seinen Gymnasialjahren unter dem Namen Gregor in das Brünner Kloster der Augustiner-Eremiten eintrat und 1869 zu seinem Abt gewählt wurde, hat bekanntlich aus einer Serie von Kreuzungsversuchen mit Erbsensorten, die sich über 5 Jahre hinzog, Gesetzmäßigkeiten der Vererbung abgeleitet, für die er eine oft gerühmte klare, mathematische Sprache fand. Diese ,Sprache‘ war möglicherweise zunächst einer der Gründe dafür, dass man ihm nicht zuhörte. Auch das Weghören kann eine Station der Sprach- und Wissenschaftsgeschichte sein. Sein Biograph Iltis berichtet: Als Mendel 1865 vor der Gesellschaft für naturwissenschaftliche Studien in Brünn seine ,Experimente in der Pflanzenkreuzung، vorträgt, verliert er die Aufmerksamkeit der meisten Zuhörer, sie schweifen $\mathrm{ab}$,bei den ziemlich schwierigen mathematischen Ableitungen, und kein einziger ist da, der so recht versteht, worauf Mendel hinaus will“. - „Fragen wurden nicht gestellt, eine Diskussion fand nicht statt“, heißt es lapidar im Protokoll (Krumbiegel 1957: 60), und Iltis vermutet, dass bei diesem Stutzen vor der „wundersamen Verknüpfung von Botanik und Mathematik“ die Erinnerung an „die Naturphilosophie der Schellingschen und Okenschen Schulen“ im Spiele war.

Spukte doch Art und Stil dieser Schule auch in den Verhandlungsbänden des Vereines herum: [...] dem weder genügend vorbereiteten, noch wohl ganz vorurteilsfreien Hörer und 
Leser mußte die „botanische Mathematik“ Mendels fremdartig genug, ja mysteriös erscheinen. (Iltis 1924: 119)

Bewirkte also eine Ausdrucksweise, die heute als einfache Statistik gelten würde, dass Mendels Vererbungslehre zu seinen Lebzeiten nicht begriffen und in ihrer Bedeutung verkannt wurde? Die Überlegung taucht in der Forschung wiederholt auf, ohne dass man hier den Hauptgrund sehen möchte (außer Iltis vgl. Krumbiegel 1957: 60; Sander 1988; Barber 1973: 213, 216).

In der Gegenwart lässt sich leichter der umgekehrte Fall vorstellen, dass nämlich ein Autor, der sich gemeinsprachlich und vormathematisch ausdrückt, wissenschaftlich nicht akzeptiert wird. Auf meine diesbezügliche Frage habe ich von zwei angesehenen Biologen zwei verschiedene Antworten erhalten. Der Freiburger Zoologe Günther Osche schreibt:

Das hängt davon ab, auf welchem Gebiet er arbeitet. Ernst Mayr als Evolutionsbiologe und Konrad Lorenz als Verhaltensforscher haben sich ausschließlich „gemeinsprachlich“ und „nicht mathematisch“ ausgedrückt und haben höchste Anerkennung gefunden, dasselbe gilt in neuerer Zeit auch für Wilson, den Begründer der modernen Soziobiologie.

Der Botaniker Burkhart Frenzel, Universität Hohenheim, antwortet dagegen:

Die Anwendung der guten, nicht alle Feinheiten bedenkenlos ausschöpfenden Gemeinsprache ist kein Hinderungsgrund der Verständigung in der Biologie. Eine Schwierigkeit liegt aber darin, daß man vielfach erst die mathematische Ausarbeitung als Beweis der Richtigkeit ansieht, wo ein einfacher Blick bereits klar und bündig die Zusammenhänge zeigt.

Recht extrem äußerte sich schon vor einiger Zeit der Berliner Physiker Ekkehard Matthias:

Ein Physiker, der sich nicht der mathematischen Formensprache bedient, ja, der seine Inhalte noch gemeinsprachlich mitteilen kann, ist im Jahr 1982 kein Physiker. - Es muß ein alter Herr sein, der auf die Vergangenheit zurückblickt. ${ }^{7}$

7 Herr Osche hat seine Antwort in einem gemeinsamen Seminar „Der Einfluß der Umgangssprache auf die wissenschaftliche Terminologie und deren Rückwirkung “ mit Freiburger Biologen im Sommersemester 1988 vorgelegt; die Antwort von Herrn Frenzel verdanke ich einem Brief vom 4.1.1989; die Äußerung von Herrn Matthias fiel in einer Diskussion am Wissenschaftskolleg zu Berlin im Winter 1981/82. 


\section{Die Anstößigkeit des Deutschen, wo Englisch erwartet wird}

Die in der Scientific Community des Westens und teilweise auch des Ostens erwartete Sprache ist seit weniger als drei Jahrzehnten Englisch. Das gilt für die Naturwissenschaften, die Medizin, teilweise auch für Psychologie und Linguistik.

Der Wechsel erscheint wie eine Umkehrung jenes folgenreichen Übergangs, der am Beginn der Neuzeit steht. Wie seinerzeit die europäischen Wissenschaften von der lingua europaea universalis zur Volkssprache übergehen, sich nationalisieren, so verabschieden sie sich seit kurzem wieder von den Nationalsprachen und gehen zur englischen Weltsprache über. Der lateinische Gelehrte bediente sich allerdings der nur für bestimmte Bereiche und Zwecke reservierten Universalsprache einer abgehobenen Schriftkultur, der heutige Wissenschaftler hat eine lebendige, mündliche wie schriftliche, in allen Bereichen gebrauchte Weltsprache zur Verfügung, die zugleich mehrfach Nationalsprache ist. Und während der erste Übergang sich in Deutschland über drei Jahrhunderte unter z. T. lebhaften Auseinandersetzungen hinzieht, vollzieht sich der zweite in drei Jahrzehnten fast lautlos; - „ja, ist dieser Übertritt denn nicht einmal ein Schwellenbewußtsein wert?“ fragte Harald Weinrich beim Konstanzer Literaturgespräch über ,Deutsch als Wissenschaftssprache“ (Weinrich 1986b: 190ff.; vgl. auch Weinrich 1986a: 18).

Der Wechsel ist bisher kaum erforscht, und ich beschränke mich im Sinne unserer Frage auf nur wenige Anmerkungen. Es ist von vornherein zu vermuten, dass besonders empfindliche Sanktionen wirksam sein müssen, wo ein so überraschend schneller Sprachwechsel vor sich geht. Wie lauten sie? Ich zitiere einige Sätze aus Tagungen, Gesprächen, Briefen:

„Die Spitzenforschung spricht englisch“, hieß ein beim Konstanzer Literaturgespräch mehrfach wiederholter Satz; er ist nicht nur ein Indikativ, sondern zugleich ein rabiater Imperativ, der einen Umkehrschluss nahelegt.

Als ich vor etwas mehr als 20 Jahren meine Dissertation in einer international führenden Zeitschrift veröffentlichte, gab es kaum eine Diskussion darüber, daß sie auf deutsch erscheinen sollte und konnte. Heute würde ich keine Originalarbeit, auf deren Inhalt ich großen Wert lege, anders als englisch publizieren, und jeder meiner Doktoranden muß und will sich mit englischen ,Papers‘ ausweisen. (Hubert Markl 1986, 20)

In Fachzeitschriften oder auf Kongressen ist Englisch „obligatorisch“. (Markl 1986: 22)

Der Zwang, die Sprache der Naturwissenschaftler zu vereinheitlichen, hat automatisch der Mehrheit das Wort gegeben; also dem Englischen. Seit dem Zweiten Weltkrieg werden Ergebnisse, je wichtiger sie sind, desto dringlicher, englisch publiziert. (Wickler).

Deutsch wird weniger verstanden und zwangsläufig weniger zitiert. Deutsch Veröffentlichtes steht immer in Gefahr, wenigstens vorerst vergessen zu werden.“(Wickler 1986: 26). 


\begin{abstract}
Man möchte doch auch im Ausland wahrgenommen werden und im Quotation Index stehen. (Ein Astrophysiker).

Haben Forschungsergebnisse überhaupt Aussicht auf internationale Beachtung, wenn sie in Zeitschriften veröffentlicht werden, die nicht im Index Medicus und im Science Citation Index berücksichtigt werden? (Lippert 1986: 40).

Und ganz mies ist, daß mancher Kollege bei Gutachten erst einmal im Quotation Index nachsieht, ob der Betreffende häufig zitiert wird. Das kann aber nur über den großen englischsprachigen Raum geschehen. (Frenzel) (Brief vom 4.1.1989).

Hochspezialisierte wissenschaftliche Zeitungen lassen sich bei Beschränkung auf den deutschen Sprachraum häufig nicht mehr kostendeckend herstellen. (Lippert 1986: 42). ${ }^{8}$
\end{abstract}

„Obligatorisch“, „muß“, „automatisch“, „dringlich“ - die Sprache des Zwangs herrscht vor. Die Überproduktion und der Wettlauf um Prioritäten, das PrestigeGefälle, der Markt und der Quotation Index haben das Anglo-Amerikanische zu einem ersten wissenschaftlichen Selektionsfaktor werden lassen.

Deutsch ist in der Scientific Community zur unerwarteten Sprache geworden; sein Gebrauch ist Grund einer Apperzeptions- und Rezeptionsverweigerung.

Ich kehre zur Ausgangsfrage zurück:

Ist die Sprache ein selbständiger Faktor der Wissenschaftsgeschichte?

Von dem Blickwinkel dieses Beitrags aus gesehen: nein. Es gibt aber eine gewisse Selbständigkeit der Sprache; diese gewisse, gewisse im Sinn von ungewisse, Selbständigkeit versuche ich in acht Punkten zu fassen.

1. „Sprache“ meint hier Sprachgebrauch. Im Sprachgebrauch der Wissenschaft oder einzelner Fächer konkretisieren sich soziale Normen, Übereinkünfte, die - vom Gebiet der internationalen Terminologie abgesehen - selten ganz starr gehandhabt werden. Sie lassen der Variabilität des Ausdrucks und der Mischung der wissenschaftlichen Diskurstypen und sogar der Sprachen einigen Spielraum.

2. Diese Normen treten oft erst in Erscheinung, wenn sie verletzt werden und die soziale Kontrolle einsetzt. In solchen Fällen gibt es die Neigung, nur noch die Sprache zu sehen, die Sprache wird zum Stein des Anstoßes, bevor der Inhalt wahrgenommen wird. Die Wahl der unerwarteten Sprache führt zur Apperzeptionsverweigerung; Apperzeptionsgewohnheit verhindert oder überblendet Rezeption.

3. Die Durchstoßung der Norm kann aber auch den umgekehrten Erfolg haben und eine neue Sprachtradition begründen. Nach einer Phase, in der man

8 Vgl. zu dem Thema dieses Abschnitts neuerdings Skudlik 1990. 
an der Sprache Anstoß genommen hat, fragt man ihren Inhalten nach und verleibt sie sich ein. (Die Wirkung der Psychoanalyse wäre dafür ein Beispiel.)

4. Für die Wahl einer wissenschaftlichen Sprache ist einerseits das intendierte Publikum - international oder national, Laie oder Forscher - verantwortlich; andererseits auch eine Auffassung von dem wissenschaftlichen Gegenstand. Im Anstoßnehmen dokumentiert sich nicht nur ein Konflikt rivalisierender Öffentlichkeiten, sondern auch das Bewusstsein, dass die Sprache an der Herstellung des wissenschaftlichen Gegenstandes beteiligt ist. Das gilt für die gegensätzlichen Diskurstypen ,Rhetorik und Poesie oder reine Wissenschaft', ,Spekulation oder Empirie‘ wie auch für den Gegensatz ,Universalsprache und Nationalsprache‘. Sie alle haben gemeinsam, dass sie, auf je verschiedene Weise, sozial verankerte Gebrauchsvarianten sind.

5. Die Entscheidung für eine fremdsprachliche Gebrauchsvariante hat allerdings die größere Folgewirkung:

An das gelehrte Latein war einerseits ein bestimmtes wissenschaftliches Paradigma, ein Denkstil, eine Spielregel der Wahrheitsfindung gebunden; andererseits stellte es die Folie dar, vor der eine deutsche Schriftkultur sich herausbildete. Die deutsche Wissenschaftssprache ist in vieler Hinsicht nicht aus der mündlichen Sprachkultur hervorgewachsen, sondern im Bruch mit ihr, als Lehnbildung der lateinischen Schriftkultur.

Auch das Anglo-Amerikanische bringt in vielen Disziplinen, man denke an die Rezeption der „modern linguistics“ in den 1960er Jahren, ein wissenschaftliches Paradigma mit; man beginnt zu erkennen, wie weit seine Wirkung auf die Gegenwartssprache reicht. Vermutlich wird es über kurz oder lang eine ähnlich lehnprägende Bedeutung für das Deutsche haben, wie das Lateinische sie einmal gehabt hat. Das müsste sich verheerend auswirken, wenn die Scientific Community sich weiterhin auf eine wissenschaftliche Stummelsprache, das sog. „bad English“, einigt.

6. Die Entscheidung für den poetischen oder streng wissenschaftlichen, bildungssprachlichen oder formalisierenden Diskurstyp ist vor allem dadurch folgenreich, dass diese Gattungen Ordnungsformen sind, von bestimmten Spielregeln geleitete Zugänge zur Realität. Die Wahl eines Sprachtyps bedeutet immer auch eine Gegenstandsbestimmung.

Auch die Wahl des Diskurstyps hat beträchtliche Konsequenzen:

Indem die Naturwissenschaften Poesie und Rhetorik zu ihren Widersachern erklären und die Tradition einer solchen Ausarbeitung der Natur ausklammern, verliert die Natur an Dimensionen, wird sie um ihre Ethik und Ästhetik verkürzt. Und auf der anderen Seite fehlt es der außerwissenschaftlichen Naturbetrachtung an Disziplinierung durch die Sache. Unsere Literatursprache hat in diesen Bezirken weiträumige blinde Felder und hinkt dem wissenschaftlichen Bewusstsein hinterher. 
Und weiter: Wenn die Naturwissenschaften die Gemeinsprache verabschieden, schwindet ihr Zusammenhang mit der allgemeinen Erfahrung, geht der gesellschaftliche Sinn ihrer Resultate $u$. U. gar nicht oder nur verspätet auf. Mit einer doppelten Verabschiedung, als Englisch und als formalisierte Sprache, verarmt die Gemeinsprache und bleibt hinter dem Erarbeiteten zurück. Wissenschaft entzieht sich der öffentlichen Phantasie und Debatte.

7. Der Wechsel der wissenschaftlichen Sprechweise ist daher nicht einfach Zeichen des Erkenntnisfortschritts, er bedeutet immer Gewinn und Verlust. Der wissenschaftliche Sprachgebrauch ist der institutionalisierte, vergegenständlichte Teil einer gesellschaftlichen Vereinbarung darüber, was als Wissenschaft zugelassen wird. Sein Wandel ist immer auch Teil einer sich wandelnden sozialen Konvention, der Übergang zu einem neuen Sprachgebrauch zieht neue Ansichten von einem Gegenstandsbereich und ein neues soziales Umfeld nach sich.

8. Normenverletzung und die ihr folgende Sanktion, bisher kaum ein Thema der Sprachwissenschaft (vgl. die Bemerkungen von Weinrich 1980: 9ff.), sind ein Indikator von Sprachgebrauchstraditionen. Wie sich eine neuere Sprachgeschichte im Spiegel der Sprachkritik schreiben ließe, so könnte man eine Geschichte der Wissenschaftssprache konzipieren, die von zeitrepräsentativen Regelverstößen und Sanktionen ausgeht. Sie könnte sich an bekannte oder unbekannte Namen heften, in jedem Fall würde sie die Geschichte im Subjekt verankern. Sie bietet die Möglichkeit, Sprachgeschichte als Geschichte konkurrierender und einander ablösender Textgattungen zu schreiben und sie dadurch gründlicher als bisher als Teil der Sozialgeschichte erkennbar werden zu lassen. 